Editorial

\title{
On the Importance of Pure Mathematics
}

\author{
Farida Kachapova \\ School of Computing and Mathematical Sciences, Faculty of Design and Creative Technology, \\ Auckland University of Technology, New Zealand
}

It seems that nowadays pure mathematics takes a back seat to applied mathematics. In many universities mathematics departments are called departments of applied mathematics, instead of just mathematics; such a "practical" name is supposed to attract more students. Also many mathematicians declare their research applied, even when it is purely mathematical. This is done in order to make the research look "practical" and "useful".

This note intends to argue with the opinion that pure mathematics is too theoretical and useless. While serious mathematicians understand the importance of pure mathematics and will probably learn nothing new from reading this article, there are still many people who need to be convinced in the importance and usefulness of pure mathematics.

Applied mathematics develops mathematical methods that are used in science, business and industry, and applies existing mathematical methods in these fields. The latter part consists of applications of mathematics; without fundamental mathematics there would be nothing to apply. The former part means developing pure mathematics while solving a particular practical problem. For example, Newton invented his calculus in order to compute the orbits of celestial objects that move according to his law of gravitation. By the 18th century calculus was established as pure mathematics, and as a pure mathematical theory calculus has many more applications than the initial application of Newton's. This illustrates the saying of a prominent British mathematician Godfrey Hardy (2012): "Pure mathematics is on the whole distinctly more useful than applied". In other words, often the usefulness of a mathematical theory is due to its abstractness and generality. For example, group theory has numerous applications in chemistry, physics and mathematics itself because it is very abstract and therefore many structures possess group properties. "There is nothing more practical than a good theory" (James C. Maxwell).

Pure mathematics studies abstract objects. However, these objects are models of real world phenomena and the results of pure mathematics turn out to be practical, though this process might take years. There is no need to contrast pure and applied mathematics; there is often overlap and mutual influence. "No human investigation can be called real science if it cannot be demonstrated mathematically" (Leonardo da Vinci). It is even truer today: Any serious research (even in art and social sciences) involves mathematics, and that mathematics would be regarded as pure mathematics in the past; an example is parametric design in architecture. All modern technology is based on pure mathematics.

ERCIM (2008) mathematics was compared to a pyramid: On the top of the pyramid are immediate applications of mathematics to our everyday life. "However the top of this pyramid would not be so high if its base were not so wide. This special feature of mathematics derives from its internal structure. A good modern application of mathematics can typically draw from differential equations, numerical analysis and linear algebra. These may very well draw from graph theory, group theory and complex analysis. These in turn rest on the firm basis of number theory, topology and geometry. Going deeper and deeper into the roots of the mathematics, one ends up with such cornerstones of logic as model theory and set theory." Purely theoretical results are necessary for building a logical mathematical theory; some of the results will have practical applications and some might not but they are still needed to support the pyramid. Fundamental areas of pure mathematics such as mathematical logic, do not have many immediate practical applications but mathematics cannot develop without them.

There are many historical examples of discoveries in pure mathematics that seemed useless at the time but had important applications years later. These are some of them.

Negative and complex numbers were regarded as absurd and useless before the 15th century. Now complex numbers are used in electrical engineering.

For about 2000 years geometers tried to deduce the fifth Euclid's axiom from the other four axioms. In 1830 Lobachevski and Bolyai proved the independence of the fifth axiom. This was a revolutionary discovery but purely theoretical until Einstein applied it in his theory of general relativity.

Graph theory has found many applications in chemistry and computer science.

The Radon transform introduced in 1917 was applied to develop Tomography in the 1960's (it was awarded Nobel prize in medicine). This is just one example of many applications of mathematics in medicine. 
Wavelet and Fourier transforms are used in computer graphic design and in medical equipment such as MRA, blood pressure monitor, and diabetes monitor.

Topology helps understanding the molecular structures (Flapan, 2000).

Algebraic Topology has found applications in data mining.

Turing's theory of computability provided the theoretical basis to computing.

Number theory in the past had been considered an example of useless mathematics. Many years later the results of number theory, in particular, the theory of prime numbers were applied to design computer security systems such as SSL connections en.wikipedia.org/wiki/Publickey_cryptography\#History. Every time we do online booking, shopping, or banking we are protected by math. We all use computers, Internet and mobile phones, and they are essentially applications of pure mathematics. Let us remember that, and respect and support pure mathematics.

Another quote from (ERCIM, 2008) emphasizes the uniqueness and importance of mathematics: “... A mathematical result is valid forever. It may fall out of fashion, or fall outside the current area of application, but even the oldest known mathematical formulae-such as that for solving quadratic equations, known 2400 years ago by Babylonians, Chinese and later the Greeks .... -are the bread and butter of present-day elementary mathematics."

\section{References}

ERCIM, 2008. Introduction to the special theme: Maths for Everyday Life. Introduction to the Special Theme, University of Helsinki, Finland.

Flapan, E., 2000. When Topology Meets Chemistry: A Topological Look at Molecular Chirality. 1st Edn., The Mathematical Association of America, ISBN10: 9780521664820 , pp: 256.

Hardy, G.H., 2012. A Mathematician's Apology. 1st Edn., Reprint Reissue, Cambridge: Cambridge University Press, ISBN-10: 110760463X, pp: 154. 